Rodrigo Dalke Meucci ${ }^{1}$

Anaclaudia Gastal Fassa ${ }^{1}$

Neice Muller Xavier Faria ${ }^{2}$

Nadia Spada Fiori ${ }^{1}$

Vanessa Iribarrem Miranda'

Deiner Resende ${ }^{1}$

\section{Limitação no trabalho por dor lombar em fumicultores do sul do Brasil}

\author{
Work limitation due to low back pain among tobacco \\ farmers in Southern Brazil
}

1 Programa de Pós-Graduação em Epidemiologia, Departamento de Medicina Social, Universidade Federal de Pelotas. Pelotas, RS, Brasil.

${ }^{2}$ Secretaria Municipal de Saúde. Bento Gonçalves, RS, Brasil.

Contato:

Rodrigo Dalke Meucci

E-mail:

rodrigodalke@gmail.com

Este artigo é parte do projeto de tese de doutoramento em andamento de Rodrigo Dalke Meucci intitulada Dor lombar em fumicultores do Sul do Brasil.

Trabalho não apresentado em reunião científica.

Apoio: Conselho Nacional de Desenvolvimento Científico e Tecnológico (CNPQ), processo no 306341/2006-9.

Os autores declaram não haver conflitos de interesse.

Recebido: 03/05/2013

Revisado: 04/04/2014

Aprovado: 07/04/2014

\section{Resumo}

Introdução: o trabalho agrícola exige esforço físico e posturas inadequadas que são de risco para dor lombar e limitação no trabalho. A dor lombar, em especial a crônica, é responsável por grande número de afastamentos do trabalho. Objetivo: avaliou-se a prevalência de limitação no trabalho por dor lombar crônica (DLC), dor lombar no último mês (DLM) e dor lombar aguda (DLAguda), bem como as tarefas que os fumicultores deixaram de fazer devido a essas patologias. Métodos: estudo transversal realizado no ano de $2011 \mathrm{com}$ uma amostra aleatória de 2469 fumicultores de São Lourenço do Sul/RS. A investigação de fatores associados à limitação por DLM foi realizada através da regressão de Poisson. Resultados: as prevalências de limitação no trabalho por DLC, DLM e DLAguda foram respectivamente, de 37,6\%, 14,4,\% e 7,8\%. Carregar folhas, empilhar lenha e colher baixeiro foram as tarefas que os fumicultores mais deixaram de fazer. Na análise ajustada, foram associadas à limitação por DLM: idade, gastos com imposto sobre a propriedade de veículos automotores (IPVA), dificuldades para pagar dívidas e problemas psiquiátricos menores. Indivíduos com DLAguda deixam de fazer as mesmas tarefas daqueles com DLC, mas estes têm muito mais limitação no trabalho.

Palavras-chave: limitação para o trabalho; dor lombar; agricultura; saúde do trabalhador; fumicultura.

\begin{abstract}
Background: farming requires high physical effort and awkward postures, which are low back pain risk factors and restrain work. Low back pain, particularly chronic pain, causes a high number of sick leaves. Objective: to assess prevalence of work limitations due to chronic low back pain (CLBP), of low back pain in the last month (LBPLM) and of acute low back pain (ALBP), as well as the tasks farmers, owing to these injuries, are not able to cope with. Methods: cross-sectional study carried out in 2011 in a random sample of Brazilian tobacco farmers (2469) from São Lourenço do Sul/RS. Factors associated to limitation originated from LBPLM were evaluated via Poisson regression. Results: prevalence of work limitation due to CLBP, LBPLM and ALBP were respectively $37.6 \%, 14.4 \%$ and $7.8 \%$. The tasks tobacco farmers most frequently had to give up were: carrying leaves, stacking firewood and harvesting bottom leaves. In the adjusted analysis, age, road tax expenses, difficulty in paying debts and minor psychiatric disorders were associated to work limitation due to LBPLM. ALBP individuals give up performing the same tasks as CLBP individuals, but CLBP individuals have higher work limitations.
\end{abstract}

Keywords: work limitation; low back pain; agriculture; occupational health; tobacco farming. 


\section{Introdução}

O trabalho agrícola é uma atividade que requer esforço físico e que demanda alto consumo de energia humana (COUTO, 1995). A literatura aponta a agricultura como um dos ramos produtivos onde o trabalhador enfrenta problemas ergonômicos que geram alto risco para o desenvolvimento de distúrbios musculoesqueléticos e incapacidade (DAVIS; KOTOWSKI, 2007; DRISCOLL et al., 2014).

O trabalho na fumicultura é artesanal e faz uso intensivo da mão de obra familiar, sendo que o cultivo do tabaco ocorre durante o ano inteiro, envolvendo cinco fases: produção das mudas, preparo do solo, transplante, tratos culturais e colheita, cura e pré-classificação (HEEMANN, 2009). A produção das mudas requer a utilização periódica de inseticidas, fungicidas e pesticidas, além da poda das mudas quando estas atingem entre 5 a 10 centímetros de altura (HEEMANN, 2009; VOGT, 1997).

Concomitantemente ao trabalho de produção das mudas, o fumicultor realiza o preparo do solo, etapa na qual o solo é lavrado, gradeado, adubado e os sulcos preparados (VOGT, 1997).

Cerca de 60 dias após a semeadura, as mudas são transplantadas através de plantadeiras manuais para a lavoura (HEEMANN, 2009; VOGT, 1997). Em até 24 horas após o transplante, é efetuada a pulverização com inseticidas (HEEMANN, 2009).

Quando a planta floresce, é feita a capação/desbrote, a qual consiste na retirada dos brotos florais. A capação é seguida pela aplicação de um antibrotante (HEEMANN, 2009).

A colheita é a etapa que mais emprega mão de obra e tem início com a retirada das folhas mais rentes ao chão, denominada colheita do baixeiro. Esta é considerada a etapa mais difícil, pois exige que o corpo fique o tempo todo encurvado (HEEMANN, 2009; PAULILO, 1987). Em função da irregularidade no desenvolvimento das plantas, cada pé é colhido entre cinco e seis vezes.

Após a colheita, as folhas são levadas para um paiol onde, de acordo com a tecnologia empregada, serão: atadas numa vara de madeira para secagem na estufa convencional; ou grampeadas em uma calha ou, ainda, colocadas soltas na estufa modelo "Loose Leaf" (BELING, 2006). Depois de cheia, a estufa é ligada e mantida em temperatura e umidade constantes, o que exige o controle permanente (HEEMANN, 2009). A necessidade de maior controle irá variar conforme o tipo de estufa, sendo muito maior no modelo convencional.

Depois de terminada a secagem, as folhas de fumo são pré-classificadas conforme seu tamanho e cor e juntadas num processo denominado manocagem. A partir daí são acondicionadas em fardos que pesam cerca de 50 quilos cada e depois armazenadas no galpão até a venda (HEEMANN, 2009).

Em todas estas fases, existem diferentes cargas de trabalho. Dentre estas, as cargas fisiológicas são problemas ergonômicos comumente relatados pelos fumicultores e podem resultar em morbidades como a dor lombar e a resultante limitação para o trabalho (FACCHINI, 1993; LAURELL; NORIEGA, 1989).

A dor lombar é uma condição extremamente comum, que acomete a maioria dos indivíduos em algum momento da vida (HOY et al., 2010; MELLOH et al., 2008). Grande parte dos casos de dor lombar tem curso benigno, sendo que a sintomatologia e a incapacidade resultantes desaparecem entre seis a oito semanas, independentemente do emprego ou não de tratamento (ANDERSSON, 1999; KERR et al., 2001; MELLOH, 2008). Estima-se que 5\% a 10\% dos casos cronificam (LOISEL et al., 2002; MELLOH, 2008), sendo estes responsáveis por mais de $60 \%$ dos custos por benefícios decorrentes do afastamento do trabalho (MAETZEL; LI, 2006).

Cerca de 30\% dos adultos portadores de dor lombar crônica relatam alguma limitação na vida diária e $25 \%$ precisam se afastar do trabalho (PICAVET; SCHOUTEN, 2003). Entre adultos com dor lombar crônica, $67 \%$ tem a capacidade para o trabalho reduzida e $45 \%$ precisam de ajuda ocasional para realizar suas atividades de vida diária (MAKELA et al., 1993).

Em agricultores, dados sobre a dor lombar e a limitação resultante são escassos. Estudos da Suécia mostram que agricultores tem maior prevalência de dor lombar em relação a indivíduos que trabalham em outros ramos produtivos. Agricultores com dor cervical/lombar em 1990-91 tiveram maior risco de limitação social/emocional em relação a controles que não eram da agricultores uma década depois (HOLMBERG et al., 2002; THELIN et al., 2008).

Muitas vezes a limitação é parcial, ou seja, o indivíduo deixa de fazer determinadas tarefas, mas continua executando outras, entretanto, os estudos não descrevem as tarefas que os agricultores deixam de fazer (ESTILL et al., 2002). Da mesma forma, não há clareza quanto a contribuição e o papel dos diferentes tipos de dor lombar, aguda e crônica, na limitação para o trabalho.

Assim, este artigo objetiva fazer uma descrição detalhada da limitação para o trabalho decorrente da dor lombar crônica (DLC), dor lombar no último mês 
(DLM) e dor lombar aguda (DLAguda) em fumicultores do sul do Brasil.

\section{Metodologia}

O estudo sobre a limitação por dor lombar entre fumicultores faz parte de uma pesquisa de delineamento transversal que avaliou diversos desfechos relacionados à saúde destes trabalhadores no município de São Lourenço do Sul, no estado do Rio Grande do Sul. A coleta de dados foi realizada no período da colheita do fumo, janeiro a março de 2011.

\section{Desfecho}

O desfecho estudado foi limitação no trabalho por dor lombar. Este desfecho foi estabelecido através da seguinte pergunta feita aos indivíduos que relataram dor lombar: "No último ano você teve que deixar de fazer atividades no trabalho por causa dessa dor na região lombar?"

A dor lombar foi caracterizada pela presença de dores nas costas no último ano, confirmando a localização da dor na coluna lombar em uma figura de uma pessoa em posição ereta, supina e dorsal com as regiões cervical, torácica e lombar pintadas em cores diferentes (MEUCCI et al., 2013; SILVA et al., 2004). A partir desta informação diferenciou-se a DLM, referente aos indivíduos que tiveram dor lombar no último mês e a DLC, estabelecida pela resposta afirmativa à presença de dor lombar contínua por três meses ou mais no último ano (FREBURGER et al., 2009). Foram considerados como portadores de DLAguda os indivíduos com DLM que não relataram DLC.

Também caracterizaram-se as atividades no trabalho da fumicultura que os indivíduos deixaram de fazer em decorrência da dor lombar (semear, fazer o transplante das mudas, capação, aplicação de agrotóxicos, colheita do baixeiro, colheita do fumo médio/ponteiro, transporte das folhas, prender folhas de fumo nas varas, passar varas com fumo, subir andaimes, cortar lenha, empilhar lenha, classificar/ fazer manocas, enfardar o fumo, empilhar/transportar fardos de fumo, operar trator).

\section{Amostra}

Os parâmetros utilizados para o cálculo da amostra estão descritos no artigo de Fassa et al., 2014. Sucintamente, de acordo com os parâmetros utilizados para o estudo da prevalência da doença da folha do tabaco verde, o tamanho amostral estimado foi de 2584 trabalhadores (FASSA et al., 2014). Para amostragem, foram obtidas na Secretaria da Fazenda de São Lourenço do Sul, as 3851 notas fiscais emitidas no ano de 2009. Considerando o tamanho amostral desejado e estimando cerca de 3 trabalhadores por propriedade, foram selecionadas, através de aleatorização simples, 1100 notas fiscais. O uso das notas fiscais garantiu que a amostra fosse representativa dos fumicultores de São Lourenço do Sul devido à obrigatoriedade da emissão de documento fiscal na venda do tabaco para a indústria. A identificação das propriedades sorteadas foi feita em conjunto com os Agentes Comunitários de Saúde da área rural do município. Em cada propriedade foram entrevistados todos os indivíduos que trabalhassem pelo menos quinze horas semanais com atividades ligadas à fumicultura.

Foram considerados inelegíveis casos em que a nota fiscal foi emitida por alguém que não era fumicultor e que na referida propriedade não havia ninguém ligado ao cultivo do fumo, assim como indivíduos que moravam na zona urbana, ou que tinham se mudado para outros municípios. Quando o indivíduo era fumicultor no ano de 2009, mas depois deixou de ser, efetuou-se a substituição pelo vizinho mais próximo que plantasse fumo. Ao término do trabalho de campo, foram identificadas 912 propriedades e entrevistados 2469 fumicultores com 18 anos ou mais de idade, totalizando 5,9\% de perdas e recusas.

Este número de entrevistados foi suficiente para o estudo da prevalência de limitação no trabalho por DLC, DLM e DLAguda, estimando-se uma margem de erro de um ponto percentual, tendo um nível de confiança de 95\%. Para o estudo dos fatores associados à limitação por DLM, o total de indivíduos com DLM foi suficiente para estimar riscos acima de 1,7 para todas as variáveis independentes de interesse, mantendo um poder estatístico mínimo de $80 \%$ e um nível de confiança de 95\%.

\section{Variáveis Independentes}

O questionário da propriedade coletou informações socioeconômicas (quantidade de fumo produzido no último ano e gastos com o Imposto sobre a Propriedade de Veículos Automotores - IPVA) para caracterização aproximada da renda e do nível econômico das propriedades, respectivamente. Também foi avaliada a dificuldade para o pagamento de dívidas através da seguinte pergunta: "Atualmente vocês estão com dificuldades para o pagamento de dívidas financeiras"?

O questionário individual avaliou as variáveis sócio-demográficas e comportamentais sexo, idade e tabagismo. Foram considerados fumantes os indivíduos que relataram fumar um ou mais cigarros por dia há mais de um mês, e ex-fumantes aqueles que tinham deixado de fumar há mais de um mês. 
A presença de problemas psiquiátricos menores foi avaliada através do questionário SRQ-20, sendo estabelecidos pontos de corte diferenciados para homens e mulheres (MARI; WILLIAMS, 1986).

A aplicação dos questionários foi realizada através de computadores de mão (Personal Digital Assistant-PDA). Todos(as) os(as) entrevistadores(as) foram submetidos(as) a treinamento e devidamente selecionados(as).

\section{Análises}

Inicialmente, realizou-se a categorização dos tipos de dor lombar em DLC, DLAguda e DLM.

Posteriormente, realizou-se uma descrição da amostra de acordo com as variáveis independentes, estratificando-se para os três tipos de dor lombar. Calculou-se a prevalência global de limitação no trabalho para a DLC, DLM e DLAguda, com os respectivos intervalos de confiança. Da mesma forma, foram calculadas as proporções de indivíduos que deixaram de fazer cada tarefa, comparando-se os tipos de dor lombar. As tarefas semear e fazer o transplante foram analisadas apenas para a DLC porque estas tarefas não são tipicamente realizadas no período em que foi realizada a coleta dos dados.

Para investigação dos fatores associados à limitação no trabalho por DLM, procedeu-se a análise através da regressão de Poisson bruta e ajustada com variância robusta para estimar as razões de prevalências (RP) e os respectivos intervalos de confiança de 95\% (IC95\%) (BARROS; HIRAKATA, 2003). As variáveis com valor $\mathrm{p} \leq 0,2$ no teste Wald de heterogeneidade ou tendência linear foram levadas para análise multivariada com seleção para trás, sendo mantidas no modelo aquelas com valor $\mathrm{p} \leq 0,2$ e consideradas associadas aquelas com valor $\mathrm{p}<0,05$ no teste de Wald. A análise multivariada seguiu um modelo hierárquico constituído por 3 níveis: no primeiro foram incluídas variáveis demográficas e socioeconômicas; no segundo a variável comportamental tabagismo e no terceiro, os problemas psiquiátricos menores.

Este estudo foi aprovado pelo Comitê de Ética em Pesquisa da Universidade Federal de Pelotas sob o parecer 11/10 e todos os entrevistados assinaram um termo de consentimento livre e esclarecido.

\section{Resultados}

Como pode ser observado na Tabela 1, do total de 2469 indivíduos entrevistados, 8,4\% (207) relataram DLC e 36,0\% (888) relataram DLM. A prevalência de DLAguda foi de $30,8 \%$ (696).
De acordo com a análise comparativa entre as variáveis independentes estratificada pelos tipos de dor lombar, verificou-se que, enquanto 37,7\% (IC95\% 31,0-44,3) dos indivíduos com DLC tinham 50 anos ou mais de idade, esta proporção foi de 26,7\% (IC95\% 23,8-29,6) para aqueles com DLM e 23,3\% (IC95\% 20,1-26,4) para os portadores de DLAguda.

Entre os indivíduos com DLC, 39,9\% (IC95\% 33,1-46,7) produziram entre 5001 a $10000 \mathrm{~kg}$ de fumo, e entre aqueles com DLM e DLAguda, esta proporção foi respectivamente de 44,7\% (IC95\% 41,4-48,0) e 45,9\% (IC95\% 42,2-49,6).

Enquanto 32,5\% (IC95\% 26,0-39,0) dos indivíduos com DLC tinham dificuldade em pagar dívidas, entre aqueles com DLAguda, esta proporção foi de 22,2\% (IC95\%19,1-25,3).

Os portadores de DLC apresentaram maior prevalência de tabagismo em relação àqueles com DLM e DLAguda, 25,6\% (IC95\% 19,6-31,6), 19,3\% (IC95\% 16,8-22,0) e 17,7\% (IC95\%14,8-20,5), respectivamente.

A prevalência de problemas psiquiátricos menores em portadores de DLC foi duas vezes maior que a dos portadores de DLAguda, 33,5\% (IC95\% 26,9$40,0)$ e $16,1 \%(13,4-18,9)$, respectivamente.

A prevalência do auto-relato de limitação no trabalho por dor lombar foi de 6,2\% (IC95\% 5,2-7,1). Entretanto, a prevalência de limitação no trabalho foi muito superior entre os indivíduos com DLC $(37,6 \%)$ em relação àqueles com DLM $(14,4 \%)$ e DLAguda $(7,8 \%)$, evidenciando que quase metade da prevalência de limitação entre indivíduos com DLM é decorrente da DLC (Tabela 2).

A proporção de indivíduos que deixaram de fazer as tarefas analisadas variou de $9 \%$ a $27,6 \%$ entre aqueles com DLC, de $2,4 \%$ a $9,1 \%$ entre aqueles com DLM e de $0,9 \%$ a $4,1 \%$ naqueles com DLAguda. Em quase todas as atividades analisadas, a prevalência de limitação no trabalho foi cerca de três vezes maior para os indivíduos com DLC em relação aos com DLM e sete vezes maior em relação àqueles com DLAguda (Tabela 3).

As tarefas que os indivíduos com DLC mais deixaram de fazer foram: transportar as folhas, $27,6 \%$ (IC95\% 21,4-33,9); fazer o transplante, 22,8\% (IC95\% 17,0-28,6); empilhar lenha, 22,2\% (IC95\% 16,3-28,1); e colher o baixeiro, 21,9\% (IC95\% 16,2$27,7)$. Os indivíduos com DLM deixaram mais frequentemente de transportar as folhas, 9,1\% (IC95\% 7,2-11,0), e empilhar lenha, 6,8\% (IC95\% 5,1-8,5). Aqueles com DLAguda deixaram mais frequentemente de: transportar as folhas, 4,1\% (IC95\% 2,65,5); colher o baixeiro, 3,6\% (IC95\% 2,2-5,0); e subir andaimes, 3,3\% (IC95\% 2,0-4,7) (Tabela 3). 
Tabela 1 Descrição da amostra de fumicultores de acordo com as variáveis independentes, estratificada por Dor lombar crônica (DLC), Dor lombar no último mês (DLM) e Dor lombar aguda (DLAguda), São Lourenço do Sul, RS, 2011

\begin{tabular}{|c|c|c|c|c|c|c|}
\hline \multirow{2}{*}{ Variável } & \multicolumn{2}{|c|}{$D L C(N=207)$} & \multicolumn{2}{|c|}{$D L M(N=888)$} & \multicolumn{2}{|c|}{ DL Aguda $(N=696)$} \\
\hline & $N$ & $\%(I C 95 \%)^{*}$ & $N$ & $\%(I C 95 \%)^{*}$ & $N$ & $\%(I C 95 \%) *$ \\
\hline \multicolumn{7}{|l|}{ Sexo } \\
\hline Masculino & 114 & $55,1(48,2-61,9)$ & 490 & $55,2(52,0-58,5)$ & 385 & $55,4(51,7-59,1)$ \\
\hline Feminino & 93 & $44,9(38,1-51,8)$ & 397 & $44,8(41,5-48,0)$ & 310 & $44,6(40,9-48,3)$ \\
\hline \multicolumn{7}{|l|}{ Idade } \\
\hline $18-29$ & 17 & $8,2(4,4-12,0)$ & 189 & $21,3(18,6-24,0)$ & 174 & $25,0(21,8-28,2)$ \\
\hline $30-39$ & 41 & $19,8(14,3-25,3)$ & 226 & $25,4(22,6-28,3)$ & 189 & $27,1(23,8-30,5)$ \\
\hline $40-49$ & 71 & $34,3(27,8-40,8)$ & 236 & $26,6(23,7-29,5)$ & 171 & $24,6(21,4-27,8)$ \\
\hline 50 ou mais & 78 & $37,7(31,0-44,3)$ & 237 & $26,7(23,8-29,6)$ & 162 & $23,3(20,1-26,4)$ \\
\hline \multicolumn{7}{|c|}{ Quantidade de Fumo Produzido (kg) } \\
\hline $1-2500$ & 14 & $6,9(3,4-10,4)$ & 63 & $7,2(5,4-8,9)$ & 50 & $7,2(5,3-9,1)$ \\
\hline 2501 a 5000 & 73 & $36,0(29,3-42,6)$ & 239 & $27,1(24,2-30,1)$ & 173 & $25,0(21,7-28,2)$ \\
\hline 5001 a 10000 & 81 & $39,9(33,1-46,7)$ & 394 & $44,7(41,4-48,0)$ & 318 & $45,9(42,2-49,6)$ \\
\hline 10001 a 36000 & 35 & $17,2(12,0-22,5)$ & 185 & $21,0(18,3-23,7)$ & 152 & $21,9(18,8-25,0)$ \\
\hline \multicolumn{7}{|c|}{ Gasto com IPVA***** $(\mathrm{R} \$)$} \\
\hline Isento & 26 & $13,2(8,4-18,0)$ & 107 & $12,4(10,2-14,7)$ & 82 & $12,1(9,6-14,6)$ \\
\hline Até 500 & 78 & $39,6(32,7-46,5)$ & 311 & $36,2(32,9-39,4)$ & 242 & $35,7(32,1-39,3)$ \\
\hline 501 a 1000 & 65 & $33,0(26,4-39,6)$ & 289 & $33,6(30,4-36,8)$ & 228 & $33,6(30,1-37,2)$ \\
\hline$>1001$ & 28 & $14,2(9,2-19,1)$ & 153 & $17,8(15,2-20,3)$ & 126 & $18,6(15,6-21,5)$ \\
\hline \multicolumn{7}{|c|}{ Dificuldade em pagar dívidas } \\
\hline Não & 137 & $67,5(61,0-74,0)$ & 667 & $75,6(72,878,5)$ & 540 & $77,8(74,7-80,9)$ \\
\hline Sim & 66 & $32,5(26,0-39,0)$ & 215 & $24,4(21,5-27,2)$ & 154 & $22,2(19,1-25,3)$ \\
\hline \multicolumn{7}{|l|}{ Tabagismo } \\
\hline Não & 128 & $61,8(55,2-68,5)$ & 576 & $64,9(61,7-68,0)$ & 458 & $65,8(62,3-69,3)$ \\
\hline Ex-fumante & 26 & $12,6(8,0-17,1)$ & 140 & $15,8(13,4-18,2)$ & 115 & $16,5(13,8-19,3)$ \\
\hline Fumante & 53 & $25,6(19,6-31,6)$ & 172 & $19,3(16,8-22,0)$ & 123 & $17,7(14,8-20,5)$ \\
\hline \multicolumn{7}{|l|}{$\mathrm{PPM}^{* * * * * *}$} \\
\hline Não & 135 & $66,5(60,0-73,0)$ & 690 & $79,9(77,3-82,7)$ & 566 & $83,9(81,1-86,6)$ \\
\hline Sim & 68 & $33,5(26,9-40,0)$ & 173 & $20,1(17,4-22,7)$ & 109 & $16,1(13,4-18,9)$ \\
\hline
\end{tabular}

*Intervalo de Confiança de 95\%.

*** Imposto sobre a propriedade de veículos automotores

***:* Problemas psiquiátricos menores 
Tabela 2 Prevalência de auto-relato de limitação no trabalho de acordo com o tipo de dor lombar, entre fumicultores (n=2469) de São Lourenço do Sul, RS, 2011

\begin{tabular}{lc}
\hline \multicolumn{1}{c}{ Tipo de dor lombar } & \%IC (95\%)* \\
\hline Dor lombar crônica - DLC & $37,6(31,0-44,2)$ \\
Dor lombar último mês - DLM & $14,4(12,1-16,7)$ \\
Dor lombar aguda - DLAguda & $7,8(5,8-9,8)$ \\
\hline * Intervalo de confiança de 95\% &
\end{tabular}

Tabela 3 Tarefas que deixou de fazer devido a Dor lombar crônica (DLC), Dor lombar no último mês (DLM) e Dor lombar aguda (DLAguda), São Lourenço do Sul - RS, 2011

\begin{tabular}{|c|c|c|c|c|c|c|}
\hline & \multicolumn{2}{|c|}{$D L C N=207$} & \multicolumn{2}{|c|}{$D L M N=888$} & \multicolumn{2}{|c|}{$D L A N=696$} \\
\hline & $N^{*}$ & $\%(I C 95 \%))^{* *}$ & $N^{*}$ & $\%(I C 95 \%)^{* * *}$ & $N^{*}$ & $\%(I C 95 \%))^{* * *}$ \\
\hline \multicolumn{7}{|c|}{ Deixou de semear } \\
\hline Não & 183 & $89,3(85,0-93,5)$ & - & & - & \\
\hline Sim & 22 & $10,7(6,5-15,0)$ & - & & - & \\
\hline \multicolumn{7}{|c|}{ Deixou de fazer o transplante } \\
\hline Não & 159 & $77,2(71,4-83,0)$ & - & & - & \\
\hline Sim & 47 & $22,8(17,0-28,6)$ & - & & - & \\
\hline \multicolumn{7}{|c|}{ Deixou de fazer a capação } \\
\hline Não & 179 & $87,3(82,7-91,9)$ & 849 & $95,8(94,5-97,1)$ & 686 & $98,1(97,1-99,1)$ \\
\hline Sim & 26 & $12,7(8,1-17,3)$ & 37 & $4,2(2,9-5,5)$ & 13 & $1,9(0,9-2,9)$ \\
\hline \multicolumn{7}{|c|}{ Deixou de aplicar agrotóxicos } \\
\hline Não & 157 & $81,4(75,8-87,0)$ & 816 & $94,4(92,9-96,0)$ & 671 & $98,1(97,1-99,1)$ \\
\hline Sim & 36 & $18,6(13,1-24,2)$ & 48 & $5,6(4,0-7,1)$ & 13 & $1,9(0,9-2,9)$ \\
\hline \multicolumn{7}{|c|}{ Deixou de colher o baixeiro } \\
\hline Não & 160 & $78,1(72,3-83,8)$ & 817 & $92,3(90,6-94,1)$ & 669 & $96,4(95,0-97,8)$ \\
\hline Sim & 45 & $21,9(16,2-27,7)$ & 68 & $7,7(5,9-9,4)$ & 25 & $3,6(2,2-5,0)$ \\
\hline \multicolumn{7}{|c|}{$\begin{array}{l}\text { Deixou de colher fumo médio/ } \\
\text { ponteiro }\end{array}$} \\
\hline Não & 175 & $84,5(79,6-89,5)$ & 847 & $95,6(94,2-97,0)$ & 684 & $98,6(97,7-99,4)$ \\
\hline Sim & 32 & $15,5(10,5-20,4)$ & 39 & $4,4(3,0-5,8)$ & 10 & $1,4(0,5-2,3)$ \\
\hline \multicolumn{7}{|c|}{ Deixou de transportar as folhas } \\
\hline Não & 144 & $72,4(66,1-78,6)$ & 796 & $90,9(89,0-92,8)$ & 664 & $95,9(94,5-97,4)$ \\
\hline Sim & 55 & $27,6(21,4-33,9)$ & 80 & $9,1(7,2-11,0)$ & 28 & $4,1(2,6-5,5)$ \\
\hline \multicolumn{7}{|c|}{$\begin{array}{l}\text { Deixou de prender as folhas de } \\
\text { fumo nas varas }\end{array}$} \\
\hline Não & 183 & $89,3(85,0-93,5)$ & 851 & $96,2(94,9-97,4)$ & 681 & $98,0(96,9-99,0)$ \\
\hline Sim & 22 & $10,7(6,5-15,0)$ & 34 & $3,8(2,6-5,1)$ & 14 & $2,0(1,0-3,1)$ \\
\hline
\end{tabular}


(...) Tabela 3 Tarefas que deixou de fazer devido a Dor lombar crônica (DLC), Dor lombar no último mês (DLM) e Dor lombar aguda (DLAguda), São Lourenço do Sul - RS, 2011

\begin{tabular}{|c|c|c|c|c|c|c|}
\hline & \multicolumn{2}{|c|}{$D L C N=207$} & \multicolumn{2}{|c|}{$D L M N=888$} & \multicolumn{2}{|c|}{$D L A N=696$} \\
\hline & $N^{*}$ & $\%(I C 95 \%)$ *** & $N^{*}$ & $\%(I C 95 \%)$ 档 & $N^{*}$ & $\%(I C 95 \%))^{* * *}$ \\
\hline \multicolumn{7}{|c|}{$\begin{array}{l}\text { Deixou de passar as varas com } \\
\text { fumo }\end{array}$} \\
\hline Não & 174 & $85,7(80,9-90,6)$ & 837 & $95,1(93,7-96,5)$ & 676 & $97,7(96,6-98,8)$ \\
\hline Sim & 29 & $14,3(9,4-19,1)$ & 43 & $4,9(3,4-6,3)$ & 16 & $2,3(1,2-3,4)$ \\
\hline \multicolumn{7}{|c|}{ Deixou de subir andaimes } \\
\hline Não & 158 & $82,3(76,8-87,7)$ & 809 & $93,6(92,0-95,3)$ & 664 & $96,7(95,3-98,0)$ \\
\hline Sim & 34 & $17,7(12,3-23,1)$ & 55 & $6,4(4,7-8,0)$ & 23 & $3,3(2,0-4,7)$ \\
\hline \multicolumn{7}{|c|}{ Deixou de cortar lenha } \\
\hline Não & 153 & $79,7(73,9-85,4)$ & 811 & $93,9(92,3-95,5)$ & 670 & $97,5(96,4-98,7)$ \\
\hline Sim & 39 & $20,3(14,6-26,1)$ & 53 & $6,1(4,5-7,7)$ & 17 & $2,5(1,3-3,6)$ \\
\hline \multicolumn{7}{|c|}{ Deixou de empilhar lenha } \\
\hline Não & 151 & $77,8(71,9-83,7)$ & 808 & $93,2(91,5-94,9)$ & 669 & $97,2(96,0-98,5)$ \\
\hline Sim & 43 & $22,2(16,3-28,1)$ & 59 & $6,8(5,1-8,5)$ & 19 & $2,8(1,5-4,0)$ \\
\hline
\end{tabular}

Deixou de classificar/fazer manocas

$\begin{array}{lcccccc}\text { Não } & 178 & 86,0(81,2-91,0) & 849 & 95,8(94,5-97,1) & 685 & 98,7(97,9-99,5) \\ \text { Sim } & 29 & 14,0(9,2-18,8) & 37 & 4,2(2,9-5,5) & 9 & 1,3(0,4-2,1)\end{array}$

Deixou de enfardar o fumo

$\begin{array}{lcccccc}\text { Não } & 180 & 87,8(83,3-92,3) & 850 & 96,4(95,1-97,6) & 683 & 98,7(97,8-99,5) \\ \text { Sim } & 25 & 12,2(7,7-16,7) & 32 & 3,6(2,4-4,9) & 9 & 1,3(0,4-2,1)\end{array}$

Deixou de empilhar/transportar fardos

$\begin{array}{lcccccc}\text { Não } & 156 & 80,8(75,2-86,4) & 816 & 94,1(92,5-95,7) & 673 & 97,7(96,6-98,8) \\ \text { Sim } & 37 & 19,2(13,6-24,8) & 51 & 5,9(4,3-7,4) & 16 & 2,3(1,2-3,4)\end{array}$

Deixou de operar trator

\begin{tabular}{ccccccc} 
Não & 172 & $91,0(87,0-95,1)$ & 837 & $97,6(96,5-98,6)$ & 677 & $99,1(98,4-99,8)$ \\
Sim & 17 & $9,0(4,9-13,1)$ & 21 & $2,4(1,4-3,5)$ & 6 & $0,9(0,2-1,60)$ \\
\hline
\end{tabular}

*O número total de indivíduos para cada atividade pode variar porque algumas atividades não eram habitualmente realizadas por todos os entrevistados.

***Intervalo de confiança de 95\%. 
De acordo com a análise ajustada para fatores associados à limitação por DLM, a idade foi diretamente associada ao desfecho, sendo maior o risco para os indivíduos mais velhos (RP 3,34; IC95\% 1,73$6,44)$. O gasto com IPVA foi inversamente associado à limitação, sendo que os indivíduos isentos do pagamento deste imposto apresentaram maior risco
(RP 2,22; IC95\% 1,03-4,79). Estar com dificuldade para pagar dívidas (RP 1,55; IC95\% 1,06-2,25) e teste positivo para distúrbios psiquiátricos menores (RP 1,95; IC95\% 1,40-2,72) também foram fatores de risco para a limitação no trabalho por DLM (Tabela 4). A correlação entre IPVA e dificuldade de pagar dívidas foi de $-0,2$.

Tabela 4 Análise bruta e ajustada da limitação para o trabalho de fumicultores por Dor lombar no último mês (DLM) de acordo com variáveis independentes, São Lourenço do Sul - RS, 2011

\begin{tabular}{|c|c|c|c|c|c|c|c|}
\hline \multirow{2}{*}{ Variável } & \multicolumn{2}{|c|}{ Bruta } & \multicolumn{2}{|c|}{ Bruta } & \multicolumn{2}{|c|}{ Ajustada } & \multirow[b]{2}{*}{$p$} \\
\hline & $\%$ & $R P \|$ & $(I C 95 \%) \dagger$ & $p$ & $R P||$ & $(I C 95 \%) \dagger$ & \\
\hline \multicolumn{8}{|l|}{ 1º Nível } \\
\hline Sexo & & & & $0,95^{*}$ & & & \\
\hline Masculino & 14,5 & 1,00 & - & & 1,00 & - & \\
\hline Feminino & 14,6 & 1,01 & $0,73-1,39$ & & - & - & \\
\hline Idade & & & & $<0,001^{\text {䊉 }}$ & & & $<0,001^{\text {粠 }}$ \\
\hline 18-29 & 5,8 & 1,00 & - & & 1,00 & - & \\
\hline $30-39$ & 13,3 & 2,28 & $1,17-4,43$ & & 2,34 & $1,19-4,36$ & \\
\hline $40-49$ & 17,8 & 3,06 & $1,62-5,78$ & & 3,02 & $1,56-5,85$ & \\
\hline 50 ou mais & 19,4 & 3,33 & $1,78-6,26$ & & 3,34 & $1,73-6,44$ & \\
\hline Quantidade de Fumo Produzido (kg) & & & & $<0,001^{* * * *}$ & & & 0,06 橉 \\
\hline 1 a 2500 & 23,8 & 1,00 & - & & 1,00 & - & \\
\hline 2501 a 5000 & 19,3 & 0,81 & $0,48-1,35$ & & 0,89 & $0,49-1,61$ & \\
\hline 5001 a 10000 & 12,7 & 0,53 & $0,32-0,59$ & & 0,70 & $0,38-1,29$ & \\
\hline 10001 a 36000 & 9,2 & 0,39 & $0,20-0,73$ & & 0,57 & $0,27-1,23$ & \\
\hline Gasto com IPVA (R\$) & & & & $<0,001^{* * * *}$ & & & $0,01^{\text {㭗 }}$ \\
\hline Isento & 22,4 & 3,43 & $1,71-6,88$ & & 2,22 & $1,03-4,79$ & \\
\hline Até 500 & 18,0 & 2,75 & $1,45-5,25$ & & 2,28 & $1,17-4,45$ & \\
\hline 501 a 1000 & 11,8 & 1,80 & $0,91-3,54$ & & 1,67 & $0,85-3,28$ & \\
\hline$>1001$ & 6,5 & 1,00 & - & & 1,00 & - & \\
\hline Dificuldade em pagar dívidas & & & & $<0,001^{*}$ & & & $0,02^{*}$ \\
\hline Não & 11,8 & 1,00 & - & & 1,00 & - & \\
\hline Sim & 22,8 & 1,92 & $1,39-2,65$ & & 1,55 & $1,06-2,25$ & \\
\hline \multicolumn{8}{|l|}{$2^{\circ}$ Nível } \\
\hline Tabagismo & & & & $0,01^{*}$ & & & 0,30 * \\
\hline Não & 12,3 & 1,00 & - & & 1,00 & - & \\
\hline Ex-fumante & 14,3 & 1,16 & $0,73-1,84$ & & 1,02 & $0,64-1,63$ & \\
\hline Fumante & 22,1 & 1,79 & $1,26-2,56$ & & 1,32 & $0,91-1,91$ & \\
\hline \multicolumn{8}{|l|}{ 3ํo Nível } \\
\hline PPM§ & & & & $<0,001^{*}$ & & & $<0,001^{*}$ \\
\hline Não & 11,6 & 1,00 & - & & 1,00 & - & \\
\hline Sim & 26,6 & 2,29 & $1,66-3,17$ & & 1,92 & $1,37-2,69$ & \\
\hline \multicolumn{8}{|l|}{ *Teste Wald de heterogeneidade } \\
\hline \multicolumn{8}{|l|}{ 楼Teste Wald de tendência } \\
\hline \multicolumn{8}{|l|}{ || Razão de Prevalência } \\
\hline$\dagger$ Intervalo de confiança de 95\% & & & & & & & \\
\hline § Problemas psiquiátricos menores & & & & & & & \\
\hline
\end{tabular}




\section{Discussão}

De acordo com os achados deste estudo, cerca de 1/5 dos indivíduos com dor lombar no "último mês" são na verdade acometidos pela dor lombar crônica. Metade da prevalência de limitação por DLM foi devida à dor crônica, sendo a limitação por DLAguda cinco vezes menor do que a devida à DLC. De modo geral, as tarefas ligadas à colheita e cura das folhas de fumo foram as atividades que os fumicultores mais deixaram de fazer em todos os tipos de dor lombar, com destaque para carregar as folhas, empilhar lenha e colher o baixeiro. Em relação aos resultados da análise ajustada, a idade foi diretamente associada à limitação no trabalho por DLM, enquanto os custos com IPVA no último ano foram inversamente associados ao desfecho. Estar com dificuldade para pagar dívidas e problemas psiquiátricos menores também foram fatores de risco para esta limitação.

Embora o desfecho deste estudo não seja a dor lombar, é importante destacar que a DLM não discrimina dor aguda e crônica. Em alguns estudos ocorre um erro de classificação porque a DLM é interpretada como sendo DLAguda. Entretanto, este viés pode comprometer a validade de estudos que ignoram a alta prevalência de dor crônica entre a DLM. Além disso, esta discussão tem implicação direta para o estudo da limitação por dor lombar, uma vez que a dor crônica foi muito mais limitante do que a aguda. A literatura discute a grande variabilidade de períodos de recordatório de dor lombar, indicando que ainda são necessários esforços no sentido de qualificar os instrumentos que avaliam esta morbidade em estudos epidemiológicos (DIONNE et al., 2008).

Os achados indicam que os portadores de DLC são obrigados a deixar de fazer, com maior frequência, aquelas atividades que resultam em maiores demandas ao sistema musculoesquelético, tais como transportar folhas, transplantar mudas, empilhar lenha e colher baixeiro. Estas são tarefas que requerem a permanência em posturas forçadas, podendo portanto, agravar a dor e/ou desconforto (DAVIS; KOTOWSKI, 2007; HEEMANN, 2009; HOLMBERG et al., 2003). A grande maioria dos indivíduos com dor aguda continua realizando todas as atividades. Entretanto, quando têm limitação, deixam de fazer as mesmas atividades que os indivíduos com dor crônica. Chama atenção que subir em andaimes é a terceira tarefa que os indivíduos com dor aguda mais deixaram de fazer, mas não aparece entre as tarefas que os indivíduos com dor crônica mais deixam de fazer. Isto pode dever-se a um viés de seleção, uma vez que apenas alguns membros da família, os mais saudáveis, são responsáveis por esta tarefa. Assim, indivíduos com dor crônica não deixam de subir em andaimes porque esta não é uma tarefa deles.
Os processos degenerativos decorrentes do envelhecimento, assim como o desgaste do trabalhador promovido pelo maior tempo de exposição às cargas de trabalho, podem explicar a associação linear direta entre a idade e a limitação no trabalho por DLM (OSBORNE et al., 2012). O envelhecimento populacional relatado em áreas rurais do sul do Brasil pode aumentar a prevalência de limitação no trabalho decorrente de distúrbios musculoesqueléticos, principalmente se não forem propostas medidas que promovam a melhoria dos processos de trabalho (CINTRA; BAZOTTI, 2012).

Indivíduos que pagam menos IPVA têm maior risco para a limitação no trabalho. Isto pode estar relacionado a menor mecanização da propriedade daqueles com menor nível econômico, implicando em maior esforço no trabalho manual. Deve-se ressaltar, contudo, que os gastos com IPVA podem não medir adequadamente a condição econômica dos fumicultores.

Estar com dificuldades para pagar dívidas, outro aspecto que pode refletir nível econômico, também foi fator de risco para a limitação no trabalho. Assim, indivíduos com menor renda e/ou com dificuldades econômicas estariam mais sujeitos a tarefas e posturas forçadas que favorecem o surgimento da limitação. Além disso, é provável que o efeito da dificuldade em pagar dívidas sobre o desfecho seja mediado pelos problemas psiquiátricos menores (CLAUSEN et al., 2013). Entretanto, ambas as associações podem estar afetadas por causalidade reversa (GORDIS, 2009).

A literatura aponta o tabagismo como fator de risco para incapacidade por dor lombar em decorrência de aterosclerose e de alterações na nutrição dos discos intervertebrais (FUJII; MATSUDAIRA, 2013; SHIRI et al., 2010). Entretanto, no presente estudo não houve associação entre esta exposição e a limitação para o trabalho. Este estudo avaliou apenas trabalhadores que não estavam afastados do trabalho, e portanto, não mediu incapacidade ou absenteísmo. Além disso, uma vez que os fumicultores estão expostos à absorção transdérmica de nicotina (ARCURY et al. 2003; FASSA et al. 2014), não havia indivíduos não expostos à nicotina no grupo de referência (não fumantes). A absorção transdérmica de nicotina pode ter efeitos sistêmicos intra-articulares similares aos do tabagismo.

É importante também considerar o efeito do trabalhador sadio nas associações examinadas. De acordo com este viés, permaneceriam trabalhando apenas os indivíduos saudáveis ou com menor limitação, sendo afastados do trabalho aqueles com limitações mais graves. Consequentemente, a prevalência do desfecho e a magnitude das associações pode estar subestimada. Entretanto, mesmo 
indivíduos com limitações mais graves podem continuar trabalhando pelo menos 15 horas semanais (critério de elegibilidade para o estudo), realizando tarefas que impliquem em menores cargas para a coluna. Na análise dos fatores associados, é preciso considerar que a DLM não diferencia sintomas agudos e crônicos.

A forma como a limitação para o trabalho foi captada, através da discriminação das tarefas que foram deixadas de fazer, constitui-se numa importante contribuição para o entendimento de que parte dos indivíduos com dor lombar não se afastam do trabalho, deixando apenas de fazer tarefas específicas, ou seja, aquelas que demandam maior sobrecarga à coluna lombar. Entretanto, o instrumento utilizado para mensurar a limitação no trabalho não é um questionário padronizado, tendo sido construído especificamente para este estudo.

Os achados deste estudo podem ser válidos não apenas para a fumicultura, mas também para outras culturas agrícolas, em especial aquelas que envolvem o trabalho familiar em lavouras temporárias, e com características semelhantes ao fumo, tais como plantas baixas e com processo de produção pouco mecanizado. Estes resultados também indicam a importância de alertar profissionais de saúde que atendem agricultores para a alta prevalência de limitação no trabalho decorrente da dor lombar. É importante capacitar estes profissionais a orientar os agricultores sobre medidas que possam prevenir a dor lombar, bem como para o correto manejo da dor aguda, especialmente com vistas a evitar a dor crônica.

\section{Contribuições de autoria}

Meucci, R. D.: realizou a revisão de literatura, supervisionou o trabalho de campo, realizou análise dos dados e redigiu o manuscrito. Fassa, A. G.: coordenou o trabalho de campo, realizou a análise e redigiu o manuscrito. Faria, N. M. X.: coordenou o trabalho de campo, realizou a análise e revisou a redação do artigo. Fiori, N. S.: supervisionou o trabalho de campo, preparou o banco de dados e revisou a redação do artigo. Miranda, V. I.: apoiou o trabalho de campo, apoiou a revisão de literatura e revisou a redação do artigo. Resende, D.: apoiou o trabalho de campo, apoiou a revisão de literatura e revisou a redação do artigo.

\section{Referências}

ANDERSSON, G. B. Epidemiological features of chronic low-back pain. Lancet, v. 354, n. 9178, p. 581$585,1999$.

ARCURY, T. A. et al. High levels of transdermal nicotine exposure produce green tobacco sickness in Latino farmworkers. Nicotine \& Tobacco Research, v. 5, n. 3, p. 315-321, 2003.

BARROS, A. J.; HIRAKATA, V. N. Alternatives for logistic regression in cross-sectional studies: an empirical comparison of models that directly estimate the prevalence ratio. BMC Medical Research Methodology, v. 3, n. 21, 2003. Disponível em: < http:// www.biomedcentral.com/1471-2288/3/21>. Acesso em: 16 jun. 2014.

BELING, R. R. A história de muita gente: um exemplo de liderança - Afubra 50 anos. Santa Cruz do Sul: Afubra, 2006.

CINTRA, A. P. U.; BAZOTTI, A. População rural, agricultura familiar e transmissão do saber na Região Sul. Caderno IPARDES, Curitiba. v. 2, n. 1, p. 80-94, 2012.

CLAUSEN, T. et al. Do self-reported psychosocial working conditions predict low back pain after adjustment for both physical work load and depressive symptoms? A prospective study among female eldercare workers. Occupational Environmental Medicine, v. 70, n. 8, p. 538-544, 2013.

COUTO, H. Ergonomia Aplicada ao Trabalho: manual técnico da máquina humana. Belo Horizonte: Ergo, 1995.

DAVIS, K. G.; KOTOWSKI, S. E. Understanding the ergonomic risk for musculoskeletal disorders in the United States agricultural sector. American Journal of Industrial Medicine, USA. v. 50, n. 7, p. 501-511, 2007.

DIONNE, C. E. et al. A consensus approach toward the standardization of back pain definitions for use in prevalence studies. Spine Journal, v. 33, n. 1, p. 95103, 2008.

DRISCOLL, T. et al. The global burden of occupationally related low back pain: estimates from the Global Burden of Disease 2010 study. Annals of the Rheumatic Diseases, v. 73, n. 6, p. 975-981, 2014.

ESTILL, C. et al. Research and dissemination needs for ergonomics in agriculture. Public Health Reports, v. 117, n. 5, p. 440-445, 2002.

FACCHINI, L. A. Uma contribuição da epidemiologia: o modelo da determinação social aplicado à saúde do trabalhador. In: BUSCHINELLI, J. T. P.; ROCHA, L. E.; RIGOTTO, R. M. Isto é trabalho de gente? Vida, doença e trabalho no Brasil. São Paulo: Vozes, 1993. p. 178-186. 
FASSA, A. G. et al. Green tobacco sickness among tobacco farmers in southern Brazil. American Journal of Industrial Medicine, v. 57, n. 6, p. 726-735, 2014. Disponível em:<http://onlinelibrary.wiley.com/ doi/10.1002/ajim.22307/abstract;jsessionid=11777CEC 9662CBC00BF52E2554225912.f04t03 > . Acesso em 04/ abr 2014.

FREBURGER, J. K. et al. The rising prevalence of chronic low back pain. Archives of Internal Medicine, v. 169, n. 3, p. 251-258, 2009.

FUJII, T.; MATSUDAIRA, K. Prevalence of low back pain and factors associated with chronic disabling back pain in Japan. European Spine Journal, v. 22, n.2, p.432-438, 2013.

GORDIS, L. Epidemiology. 4. ed. Philadelphia: Elsevier, 2009.

HEEMANN, F. O Cultivo do Fumo e Condições de Saúde e Segurança dos Trabalhadores Rurais. 2009. 171 f. Dissertação (Mestrado em Engenharia de Produção)-Universidade Federal do Rio Grande do Sul, Porto Alegre, 2009.

HOLMBERG, S. et al. Musculoskeletal symptoms among farmers and non-farmers: a population-based study. International Journal of Occupational and Environmental Health, v. 8, p. 339-345, 2002.

HOLMBERG, S. et al. The impact of physical work exposure on musculoskeletal symptoms among farmers and rural non-farmers. Annals of Agricultural and Environmental Medicine, v. 10, p. 179-184, 2003.

HOY, D. et al. The Epidemiology of low back pain. Best Practice \& Research Clinical Rheumatology, v. 24 n. 6, p. 769-781, 2010.

KERR, M. S. et al. Biomechanical and psychosocial risk factors for low back pain at work. American Journal of Public Health, v. 91, n. 7, p. 1069-1075, 2001.

LAURELL, A. C.; NORIEGA, M. Processo de produção e saúde: trabalho e desgaste operário. São Paulo: Hucitec, 1989.

LOISEL, P. et al. Cost-benefit and cost-effectiveness analysis of a disability prevention model for back pain management: a six year follow up study. Occupational Environmental Medicine, v. 59, p. 807-815, 2002.
MAETZEL, A.; LI, L. The economic burden of low back pain: a review of studies published between 1996 and 2001. Best Practice \& Research Clinical Rheumatology, v. 16, n. 1, p. 23-30, 2006.

MAKELA, M. et al. Musculoskeletal disorders as determinants of disability in Finns aged 30 years or more. Journal of Clinical Epidemiology, v. 46, n. 6, p. 549-559, 1993.

MARI, J.; WILLIAMS, P. A validity of a psychiatric screening questionnaire (SRQ-20) in primary care in the city of Sao Paulo. British Journal of Psychiatry, v. 148, p. 23-26, 1986.

MELLOH, M. et al. Differences across health care systems in outcome and cost-utility of surgical and conservative treatment of chronic low back pain: a study protocol. BMC Musculoskeletal Disorders, v. 9, n. 81, 2008.

MEUCCI, R.D. et al. Increase of chronic low back pain prevalence in a medium-sized city of southern Brazil. BMC Musculoskeletal Disorders, v. 14, n. 155, 2013.

OSBORNE, A. et al. Risk factors for musculoskeletal disorders among farm owners and farm workers: a systematic review. American Journal of Industrial Medicine, v. 55, n. 4, p. 376-389, 2012.

PAULILO, M. I. S. O Peso do Trabalho Leve. Revista Ciência Hoje, Rio de Janeiro, v. 5, n. 28, p. 64-70, 1987.

PICAVET, H. S.; SCHOUTEN, J. S. Musculoskeletal pain in the Netherlands: prevalences, consequences and risk groups, the DMC(3)-study. Pain, v. 102, n. 1-2, p. 167-178, 2003.

SHIRI, R. et al. The association between smoking and low back pain: a meta-analysis. American Journal of Medicine, v.123, n. 1, p. 87.e7-87.e35, 2010.

SILVA, M. C. et al. Chronic low back pain in a Southern Brazilian adult population: prevalence and associated factors. Cadernos de Saúde Pública, Rio de Janeiro, v. 20, n. 2, p. 377-385, 2004.

THELIN, A. et al. Functioning in neck and low back pain from a 12-year perspective: a prospective population-based study. Journal of Rehabilitation Medicine, v. 40, n.7, p. 555-561, 2008.

VOGT, O. P. A produção de fumo em Santa Cruz do Sul, RS: 1849-1993. Santa Cruz do Sul: Edunisc, 1997. 